\title{
Computing Lifetimes for Battery-Powered Devices
}

\author{
Marijn Jongerden and Boudewijn Haverkort
}

\begin{abstract}
The battery lifetime of mobile devices depends on the usage pattern of the battery, next to the discharge rate and the battery capacity. Therefore, it is important to include the usage pattern in battery lifetime computations. We do this by combining a stochastic workload, modeled as a continuous-time Markov model, with a well-known battery model. For this combined model, we provide new algorithms to efficiently compute the expected lifetime and the distribution and expected value of the delivered charge.
\end{abstract}

\section{Introduction}

The usage of wireless devices like cell phones, laptop computers or wireless sensors is often limited by the lifetime of the included batteries. The lifetime naturally depends on the capacity of the battery and the rate at which it is discharged. However, it also depends on the discharge pattern. When a battery is continuously discharged, a high current will cause it to provide less energy until the end of its lifetime than a low current. This is the so-called rate-capacity effect. On the other hand, during periods of low or no current the battery can recover partly. This is the so-called recovery-effect. To properly model the impact of the usage pattern on the battery, one has to combine a workload model with a battery model.

We combine the Kinetic Battery Model (KiBaM) [4], which is the simplest model that includes both the above effects, with a continuous-time Markov model to describe the usage pattern of the device, that is, its workload.

Marijn Jongerden

University of Twente, Enschede, The Netherlands e-mail: jongerdenmr@ewi.utwente.nl

Boudewijn Haverkort

Embedded Systems Institute, Eindhoven, The Netherlands e-mail: boudewijn.haverkort@esi.nl

University of Twente, CTIT, Enschede, The Netherlands, e-mail: brh@ewi.utwente.nl 
In [1] we have proposed this model to compute battery lifetime distributions. Here we extend our analysis in order to efficiently compute the distribution of the total charge delivered by the batteries, as well as the expected battery lifetime and the expected charge delivered. The details of this analysis are given in [3].

\section{Kinetic battery model}

We use the Kinetic Battery Model (KiBaM) [4] to model the battery. This model is the simplest model that includes the two important non-linear battery properties, the rate-capacity effect and the recovery effect [2].

In the KiBaM the battery charge is distributed over two wells: the availablecharge well and the bound-charge well (cf. Figure 1(a)). A fraction $c$ of the total capacity is put in the available charge well, and a fraction $1-c$ in the bound charge well. The available charge well supplies electrons directly to the load $(i(t))$, whereas the bound-charge well supplies electrons only to the available-charge well. The charge flows from the bound charge well to the available charge well through a "valve" with fixed conductance, $k$. Next to this parameter, the rate at which charge flows between the wells depends on the height difference between the two wells. The heights of the two wells are given by: $h_{1}=\frac{y_{1}}{c}$ and $h_{2}=\frac{y_{2}}{1-c}$. The change of the charge in both wells is given by the following system of differential equations:

$$
\left\{\begin{array}{l}
\frac{d y_{1}}{d t}=-i(t)+k\left(h_{2}-h_{1}\right), \\
\frac{d y_{2}}{d t}=-k\left(h_{2}-h_{1}\right),
\end{array}\right.
$$

with initial conditions $y_{1}(0)=c \cdot C$ and $y_{2}(0)=(1-c) \cdot C$, where $C$ is the total battery capacity. The battery is considered empty as soon as there is no charge left in the available charge well, that is, as soon as $y_{1}=0$.

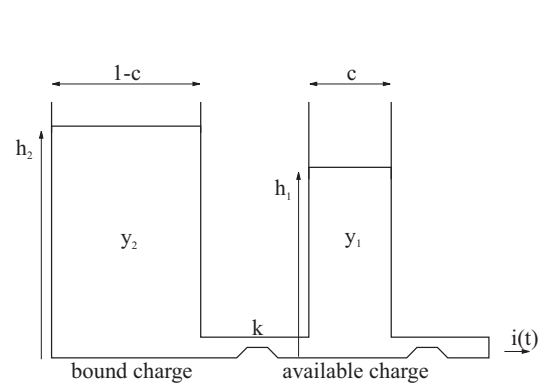

(a) Kinetic Battery Model

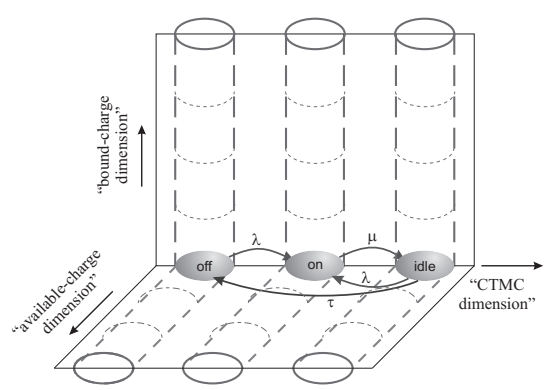

(b) MRKiBaM

Fig. 1 The two well Kinetic Battery Model and the Markov Reward KiBaM 


\section{Markov Reward KiBaM}

\subsection{Introduction}

We combine the KiBaM with a stochastic workload, modeled as a continuous-time Markov chain (CTMC), creating a Markov reward model (MRM). Each state in the CTMC represents a mode in which the device can be used, with its own discharge current. Mode switches are represented by the transitions between the states in the Markov chain. In combining the CTMC with the KiBaM, the differential equations of the KiBaM are integrated into the Markov model as accumulated rewards, which represent the levels of charge in the two charge wells. A schemetic picture of the combined model is given in Figure 1(b). The first accumulated reward $Y_{1}(t)$ represents the available-charge well, the second accumulated reward $Y_{2}(t)$ represents the bound-charge well. The corresponding rates are derived from the KiBaM differential equations (1). Let $I_{i}$ be the energy consumption rate in a state $i \in S$. The first reward rate then is

$$
r_{i, 1}\left(y_{1}, y_{2}\right)= \begin{cases}-I_{i}+k \cdot\left(\frac{y_{2}}{1-c}-\frac{y_{1}}{c}\right), & \frac{y_{2}}{1-c}>\frac{y_{1}}{c}>0, \\ 0, & \text { otherwise },\end{cases}
$$

and the second reward rate is

$$
r_{i, 2}\left(y_{1}, y_{2}\right)= \begin{cases}-k \cdot\left(\frac{y_{2}}{1-c}-\frac{y_{1}}{c}\right) & \frac{y_{2}}{1-c}>\frac{y_{1}}{c}>0, \\ 0, & \text { otherwise }\end{cases}
$$

The interesting question for battery-powered devices is: "When does the battery get empty?" For the Markov Reward KiBaM model, the battery is empty at time $t$ if the available-charge well is empty, that is, if the accumulated reward $Y_{1}(t)=0$. Since the accumulated reward $Y_{1}(t)$ is a random variable, we can only indicate the probability that the battery is empty at time $t$ :

$$
\mathbb{P}\{\text { battery empty at time } t\}=\mathbb{P}\left\{Y_{1}(t)=0\right\}
$$

The lifetime $L$ of a battery is the instant the battery gets empty for the first time, $L=\min \left\{t \mid Y_{1}(t)=0\right\}$.

\subsection{Battery lifetime}

In [1] we showed that one can approximate the MRM with a CTMC by applying a discretization to the accumulated reward levels. In fact, we approximate the continuous accumulated reward growth with a discrete stochastic equivalent in which the accumulated reward is regarded as a discrete Erlang-distributed random variable. This CTMC approximation provides in fact a phase-type distribution for a given workload model. Its absorbing states are the ones where the battery is per- 
ceived empty, that is, where the available charge $Y_{1}$ reaches zero. Such state is of the form $\left(i, 0, j_{2}\right)$, where $i$ is an original MRM state, $j_{1}=0$ represents the empty available-charge well, and $j_{2}$ is the discretized level of the charge remaining in the bound-charge well.

The generator matrix of this new CTMC $\mathbf{Q}^{*}$ can be arranged in such a way that

$$
\mathbf{Q}^{*}=\left(\begin{array}{cc}
\mathbf{0} & \mathbf{0} \\
\mathbf{T}^{0} & \mathbf{T}
\end{array}\right),
$$

where $\mathbf{T}$ contains the rates of transitions between non-absorbing states, and $\mathbf{T}^{\mathbf{0}}$ is the matrix with the rates from each non-absorbing state to the absorbing states, which indicate that the battery is empty. If we merge all absorbing states into one, the generator matrix reduces to:

$$
\mathbf{Q}^{*}=\left(\begin{array}{cc}
0 & 0 \\
\underline{T}^{0} & \mathbf{T}
\end{array}\right),
$$

where $\underline{T}^{0}$ is a column vector with the cumulative rates to the absorbing states. The represented phase-type distribution is the approximate distribution for the random variable describing the time it takes for the battery to be emptied.

The expected value of a random variable $L$ having a phase-type distribution is described by $\mathbb{E}[L]=-\underline{\alpha} \mathbf{T}^{-1} \underline{1}$, where $\alpha$ is the initial distribution, and $\underline{1}$ is a column vector of appropriate size with each element equal to one. Thus, if we solve the system of linear equations $\underline{x} \mathbf{T}=-\underline{\alpha}$, we have that $\mathbb{E}[L]=\sum_{i} x_{i}$. Using this approach we can approximate the expected battery lifetime for a given workload.

\subsection{Delivered charge}

The amount of charge that is actually delivered by the battery depends on the workload. When we look at the absorbing states of the approximating CTMC, we see that if the CTMC ends up in a state $s_{a}=\left(i, 0, j_{2}\right)$, it means that the delivered charge is approximately $C-j_{2} \Delta$, where $\Delta$ is step size of the discretization that is applied to the charge. We can thus compute approximations to the distribution and expected value of the delivered charge.

Since for these computations the time until absorption is not important, it suffices to consider the embedded discrete-time Markov chain with probability matrix $\mathbf{P}^{*}$, where

$$
P_{s, s^{\prime}}^{*}= \begin{cases}1, & \text { if } s=s^{\prime} \text { and } Q_{s, s}^{*}=0, \\ \frac{Q_{s, s^{\prime}}^{*}}{-Q_{s, s}^{*}}, & \text { if } s \neq s^{\prime} \text { and } Q_{s, s}^{*} \neq 0, \\ 0, & \text { elsewhere. }\end{cases}
$$

Following the notation introduced for phase-type distributions we can arrange $\mathbf{P}^{*}$ such that

$$
\mathbf{P}^{*}=\left(\begin{array}{cc}
\mathbf{I} & \mathbf{0} \\
\mathbf{R}^{0} & \mathbf{R}
\end{array}\right)
$$


The probability $A_{s, s_{a}}$ to end in a certain absorbing state $s_{a}$, having started in state $s$ is determined by the following system of linear equations:

$$
A_{s, s_{a}}= \begin{cases}1, & \text { if } s=s_{a} \\ \sum_{z} P_{s, z}^{*} A_{z, s_{a}}, & \text { otherwise. }\end{cases}
$$

If $\mathbf{B}$ is the matrix consisting of the values $A_{s, s_{a}}$ where $s$ is a transient state, this system of linear equations can be written as:

$$
\mathbf{R B}+\mathbf{R}^{0} \mathbf{I}=\mathbf{B} \quad \text { or } \quad(\mathbf{I}-\mathbf{R}) \mathbf{B}=\mathbf{R}^{\mathbf{0}} .
$$

This system can be solved for one column of $\mathbf{R}^{\mathbf{0}}$ at a time using standard solution algorithms [5]. The complete matrix $\mathbf{A}$ is obtained by extending the computed matrix $\mathbf{B}$ to include also the absorbing states as initial states:

$$
\mathbf{A}=\left(\begin{array}{l}
\mathbf{I} \\
\mathbf{B}
\end{array}\right)
$$

Multiplying the initial distribution vector $\underline{\alpha}$ with $\mathbf{A}$ gives the probability distribution $\underline{a}$ to end up in the different absorbing states, i.e., $\underline{a}=\underline{\alpha} \mathbf{A}$. The element $a_{\left(i, 0, j_{2}\right)}$ denotes the probability that the battery gets empty with a residual charge of $j_{2} \Delta$, and thus having delivered a charge of $C-j_{2} \Delta$. In doing so, we obtain the distribution of the delivered charge. The expected delivered charge $\mathbb{E}[d C]$ is given by:

$$
\mathbb{E}[d C]=C-\sum_{\substack{\left(i, 0, j_{2}\right) \\ \text { is absorbing }}} j_{2} \Delta a_{\left(i, 0, j_{2}\right)} .
$$

\section{Results}

For the results we use the simple workload model and battery that were also used in [1]. The simple workload model consists of three states, as depicted in Figure 1(b). Initially, the model is in idle state. With rate $\lambda=2$ per hour the model moves into the on state. On average, the model spends 10 minutes in the on state, resulting in a outgoing rate of $\mu=6$ per hour. From the idle state the device can also move into a power-saving off state, this is done - on average - once per hour $(\tau=1)$. The power-consumption rate is low when idling $\left(I_{0}=8 \mathrm{~mA}\right)$, it is high when sending data $\left(I_{1}=200 \mathrm{~mA}\right)$ and negligible in the sleep state $\left(I_{2}=0 \mathrm{~mA}\right)$.

In Figure 2, distributions of the battery lifetime and delivered charge are given for various values of the discretization parameter $\Delta$, and compared to the distributions obtained by simulation. The simulation distributions are based on 10000 runs.

In Figure 2(a) we see that the lifetime distribution is well approached already with $\Delta=10 \mathrm{mAh}$; for the delivered charge distribution $\Delta$ has to be decreased to $2 \mathrm{mAh}$ to obtain a good approximation, cf. Figure 2(b). 


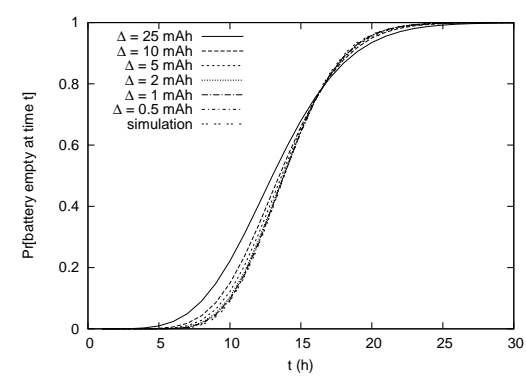

(a) lifetime distribution

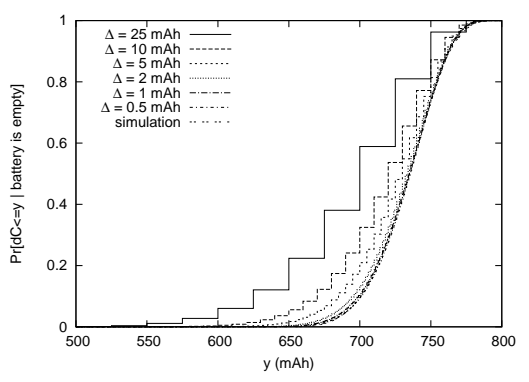

(b) delivered charge distribution

Fig. 2 Battery lifetime and delivered charge distribution for a simple workload model

Next to the distributions also the expected values of the battery lifetime and the delivered charge have been computed. For both the expected lifetime and the expected delivered charge the results are similar. The expected values for $\Delta=0.5 \mathrm{mAh}$ are $1 \%$ lower than the averages obtained from the simulation.

More results can be found in [3], where the simple model is compared with a more complex burst model, and the difference in lifetime and delivered charge between the two models is discussed.

\section{Conclusion}

We presented a new approach to compute distributions and expected values of both battery lifetime and delivered charge for random workloads. This is an extension to the work presented in [1], where only the lifetime distribution was computed. The results for a simple workload model show that the approach leads to a good approximation for both distributions and expected values.

\section{References}

1. L. Cloth, B. R. Haverkort, and M. R. Jongerden. Computing battery lifetime distributions. In Proceedings of the 37th Annual IEEE/IFIP International Conference on Dependable Systems and Networks (DSN '07), pages 780-789. IEEE Computer Society Press, 2007.

2. M. R. Jongerden and B. R. Haverkort. Which battery model to use? IET Software, 3(6):445457, December 2010.

3. Marijn Jongerden. Model-Based energy analysis of battery powered systems. $\mathrm{PhD}$ thesis, University of Twente, expected dec 2010.

4. J.F. Manwell and J.G. McGowan. Lead acid battery storage model for hybrid energy systems. Solar Energy, 50:399-405, 1993.

5. William J. Stewart. Introduction to the Numerical Solution of Markov Chains. 1994. 